http://jmscr.igmpublication.org/home/ ISSN (e)-2347-176x ISSN (p) 2455-0450 crossref DOI: https://dx.doi.org/10.18535/jmscr/v8i8.39

\title{
The added value of digital breast tomosynthesis over digital mammography in the assessment of BIRADS 0 and 3 breast lesions
}

\author{
Authors \\ Dr Sapna Patel ${ }^{1}$, Dr Sanjiv Patel $^{2}$, Dr Darshan Thummar ${ }^{3}$, Dr Divya Patel ${ }^{4}$ \\ ${ }^{1,2}$ Assi Prof, Dept of Radiology, GCRI \\ ${ }^{3,4}$ Resident Doctor, GCRI
}

\begin{abstract}
Background: The purpose of this study is to assess the role of 3-dimensional breast tomosynthesis in correctly diagnosing breast imaging-reporting and data system (BIRADS) 3 and 0 lesions.

Methods: A prospective study for mammographic cases referred to our radiology unit included 225 patients (out of 1425 patients) who were advised screening mammography during the period from January 2019 to December 2019.

The study was performed in 200 cases, detecting 131 BIRADS 3 and 0 lesions. All mammograms were classified as BIRADS 1-5 category after 3D DBT. All breast lesions that were upgraded by $3 D D B T$ were either aspirated, biopsied, or surgically removed, and were followed-up.

Results: $77.8 \%$ of BIRADS 3 and 0 lesions detected by 2D digital mammography (102/131) changed their category after $3 D$ DBT and $22.2 \%$ (29/131) of digital mammography assigned BIRADS 3 and 0 lesions did not change after $3 D$ DBT.

$77.8 \%$ percent of the lesions changed their BIRADS system classification following DBT:

1. 3D DBT upstaged to BIRADS 4 and 5 in 34 lesions representing $15.1 \%$ of the cases.

2. $3 D$ DBT down staged to BIRADS 1 and 2 in 68 lesions representing $51.9 \%$ of the cases.

We performed a biopsy in all suspected findings on tomosynthesis. Of 225 patients, 60 were proven to have malignancy. Of this 60 patients, 46 patients had a single lesion, 6 had multifocal disease, and 8 had multicentric disease.

Conclusion: 3D DBT significantly reduced the need for additional mammographic views and frequent follow-up studies as it gave better characterization for all BIRADS 3 and 0 lesions. 3D DBT did not show any false-negative results in this study and it did not miss any cancers. In addition, reduction in false positive results reduced the stress levels in women. Therefore, 3D digital breast tomosynthesis should be applied in the diagnostic algorithm in patients with mammography detected BIRADS 3 and 0 lesions.

Keywords: BI-RADS classification; Digital mammography; Tomosynthesis.
\end{abstract}

\section{Introduction}

Breast cancer is the most common malignancy in women throughout the world and thus, screening of breast with recognition of the lesion at early stage has become an important health issue.
Mammography has been used as breast cancer screening tool till date despite its well-known limitations.

The known lack of sensitivity isrelated to the 2dimensional (2D) format of conventional imaging of the 3-dimensional (3D) breast volume. Since 
some breast cancers have the same X-ray attenuation as normal breast tissue, clinically relevant malignancies may be obscured by overlapping normal tissue. In contrast, complex areas of normal tissue may be perceived as suspicious. The limitations of $2 \mathrm{D}$ breast imaging leads to low sensitivity in detecting certain cancers and high false-positive recall rates ${ }^{[1]}$.

Digital breast tomosynthesis (DBT) is a new imaging modality for improving the detection of breast cancer. The main advantage of tomosynthesis is its ability to select discrete tissue planes. As it provided better detection of the abnormalities especially in females with dense breast and confident diagnosis of benign lesions resulted in reduction in number of the recalled cases and negative biopsies, as well as it enabled visualization of cancers not visualized by conventional mammography ${ }^{[1]}$.

In this study, we mainly focused on added value of DBT in proper categorisation of DM assigned indeterminate BI-RADS categories, because reducing BI-RADS 0 and 3 has pivotal implications for patient care.

\section{Aim of the Study}

The purpose of this study is to assess the role of 3dimensional breast tomosynthesis in correctly diagnosing breast imaging-reporting and data system (BIRADS) 3 and 0 lesions.

\section{Methods}

\section{Patients}

A prospective study for mammographic cases referred to our radiology unit included 225 patients (out of 1425 patients) who were advised screening mammography during the period from January 2019 to December 2019.

The study was performed in 200 cases, detecting 131 BIRADS 3 and 0 lesions. All mammograms were classified as BIRADS1-5 category after 3D DBT. A complementary ultrasound examination was performed for all cases to confirm or exclude mammographically identified abnormalities using high frequency probe. All breast lesions that were upgraded by 3D DBT were either aspirated, biopsied, or surgically removed, and were followed-up.

\section{Inclusion Criteria}

Each patient underwent clinical breast examination, DM and DBT; the medical history was also evaluated. Women presenting with abnormal findings by conventional mammography and assigned BIRADS 3 and 0 lesions.

\section{Equipments}

Mammographic examination was performed using Siemens Mammomat 1000 full-field digital mammography machine with Siemens Mammomat Inspiration 3D digital breast tomosynthesis machine, with the use of software that allowed synthetic 2D mammographic images to be reconstructed from 3D acquisitions.

\section{Technique of full field digital mammography}

Standard views: Medio-lateral-oblique and Cranio-caudal views were taken for all patients.

\section{Technique of 3D tomosynthesis:}

For 3D digital tomosynthesis, two views (MLO and CC) were obtained.

Siemens' breast tomosynthesis unit acquires 25 images during a continuous scan, while the X-ray source rotates along a predefined arc of $\pm 25^{\circ}$ relative to the $0^{\circ}$ position around the compressed breast, with a $2^{\circ}$ angle increment per image. Images are reconstructed using filtered back projection in order to provide sections parallel to the breast support. It provided synthetic $2 \mathrm{~d}$ mammographic images which were reconstructed from $3 \mathrm{D}$ acquisitions ${ }^{[4]}$.

\section{Image analysis and interpretation of mammography and 3D digital tomosynthesis} The reader independently read 2DM and 3DBT. Images were evaluated in 2 steps: DM first (DBT examinations were not available to the radiologist at this time to avoid clinical decision making being affected), and after a period of 1 week, evaluation of DBT views was done. 
The lesions were analysed using standardized morphological descriptors:

1. Breast density was assessed for each patient according to ACR guidelines for breast composition $^{[2]}$.

2. Each lesion was evaluated regarding site and type (mass, focal asymmetry \pm calcifications and size).

3. BIRADS for the lesions were assigned according to the mammography ACR BIRADS 2013 lexicon morphology descriptors ${ }^{[2]}$ :

- Mass lesions: shape, margin, density, and size

- Asymmetry: simple, focal, global, or developing

- Calcifications: morphology and distribution.

- At the end of each reading step, the radiologist gave a probability of breast lesion being benign or malignant using the ACR BIRADS - 2013 classification $(0-5)^{[2]}$ and hence determined the need for further diagnostic investigations. Patients with BIRADS 0, 3, 4 and 5 were recalled for further work-up.

\section{Birads interpretation and patient management}

\begin{tabular}{|l|l|}
\hline Category & Management \\
\hline Birads 0 and 3 & $\begin{array}{l}\text { Recall for additional imaging and/or } \\
\text { await prior examinations }\end{array}$ \\
\hline Birads 1 and 2 & Routine screening \\
\hline Birads 4 and 5 & Tissue diagnosis \\
\hline
\end{tabular}

\section{Results}

Table 1: BIRADS Categorisation of the Breasts Lesions in 225 Patients on DM and DBT

\begin{tabular}{|l|c|c|}
\hline & DM $(\mathrm{n}-225)$ & DBT $(\mathrm{n}-225)$ \\
\hline BIRADS 1 and 2 & $67(29.7 \%)$ & $135(60 \%)$ \\
\hline BIRADS 0 and 3 & $131(58 \%)$ & $29(12.8 \%)$ \\
\hline BIRADS 4 and 5 & $27(12 \%)$ & $61(27.1 \%)$ \\
\hline
\end{tabular}

Table 2: Correlation of the Mammographically (DM) Detected Lesions with USG/ Histo-Pathology

\begin{tabular}{|l|c|c|c|c|c|}
\hline BIRADS & $\mathbf{N = 2 2 5}$ & \multicolumn{2}{|c|}{ USG } & \multicolumn{2}{c|}{ HPE } \\
\hline & & BENIGN & SUSPICIOUS & BENIGN & MALIGANT \\
\hline 1,2 & 67 & 63 & 4 & 4 & $X$ \\
\hline 0,3 & 131 & 68 & 34 & 20 & 14 \\
\hline 4,5 & 27 & 0 & 27 & 6 & 21 \\
\hline
\end{tabular}

$\mathrm{X}=$ not applicable
A prospective study for mammographic cases referred to our radiology unit included 239 lesions detected in225patients that were performed during the period from January 2019 to December 2019.

\section{Age}

Patients' ages ranged from 27 to 82 years with mean age $58 \pm 15$

\section{Breast density ACR scoring}

According to the 2013 American College of Radiology BIRADS lexicon, classification of breast density composition categories are ${ }^{[2]}$ :

- Almost entirely fatty breasts, ACR a.

- Breasts with scattered areas of fibro glandular density, ACR b.

- Breasts which are heterogeneously dense, which may obscure small masses, ACR c.

- Breasts which are extremely dense, which lowers the sensitivity of mammography, ACR d.

Breast density distribution of the patients ( $\mathrm{n}$ 225), According to the ACR BIRADS lexicon:

- 13/225 (5.77\%) lesions were assigned an ACR score of "a".

- 56/225 (24.88\%) lesions were assigned an ACR score of "b".

- 134/225 (59.55\%) lesions were assigned an ACR score of "c".

- 22/225 (9.7\%) were assigned an ACR score of "d". 
Out of 27 lesions assigned BIRADS 4 and 5 on DM, 5 lesions turned out to be benign on HPE out of which 4 were fibrotic scars and 2 cases of old abscess.

Table 3: Correlation of the Tomosynthesis (DBT) Detected Lesions with USG/ Histo-Pathology

\begin{tabular}{|l|c|c|c|c|c|}
\hline \multirow{2}{*}{ BIRADS } & \multirow{2}{*}{$\mathbf{2} \mathbf{2 2 5}$} & \multicolumn{2}{|c|}{ USG } & \multicolumn{2}{c|}{ HPE } \\
\cline { 3 - 6 } & & BENIGN & SUSPICIOUS & BENIGN & MALIGANT \\
\hline 1,2 & 135 & 132 & 3 & 3 & $\mathrm{X}$ \\
\hline 0,3 & 29 & 26 & 3 & 3 & $\mathrm{X}$ \\
\hline 4,5 & 61 & 0 & 61 & 4 & 57 \\
\hline
\end{tabular}

Out of 61 lesions assigned BIRADS 4 and 5 on DM, 4 lesions turned out to be benign on HPE out of which 4 were fibrotic scars.

\section{Cases}

\section{Figure 1:}

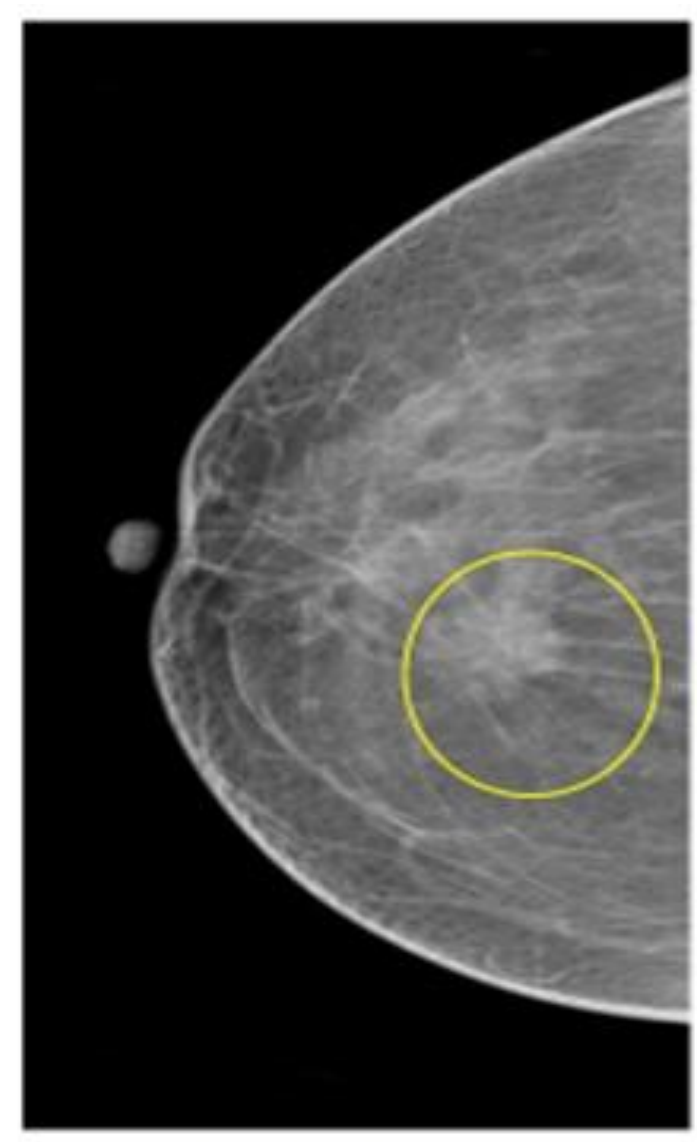

FIGURE A - DM

Right breast - CC view

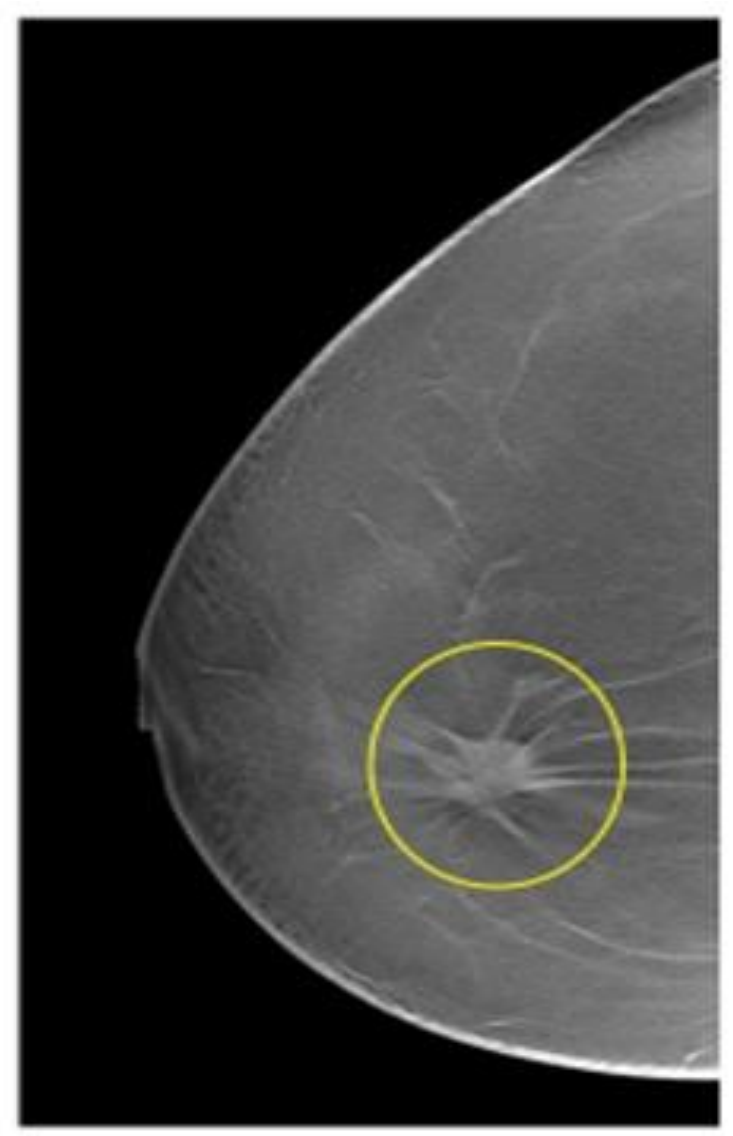

FIGURE B - DBT

Right breast $-\mathrm{CC}$ view 44-year-old female mammography CC views: ACR B.

DM: Right upper central region small oval illdefined mass lesion - BIRADS 3.

DBT: The margin of the lesion appeared spiculated on 3D digital tomosynthesis images (BIRADS 4). The tomosynthesis has better margin characterization, which easily detected the spiculated margin of this lesion and upgraded the BIRADS category from 3 to 4 . Ultrasoundconfirmed speculated borders. Pathologically proved to be ductal carcinoma. 
Figure 2

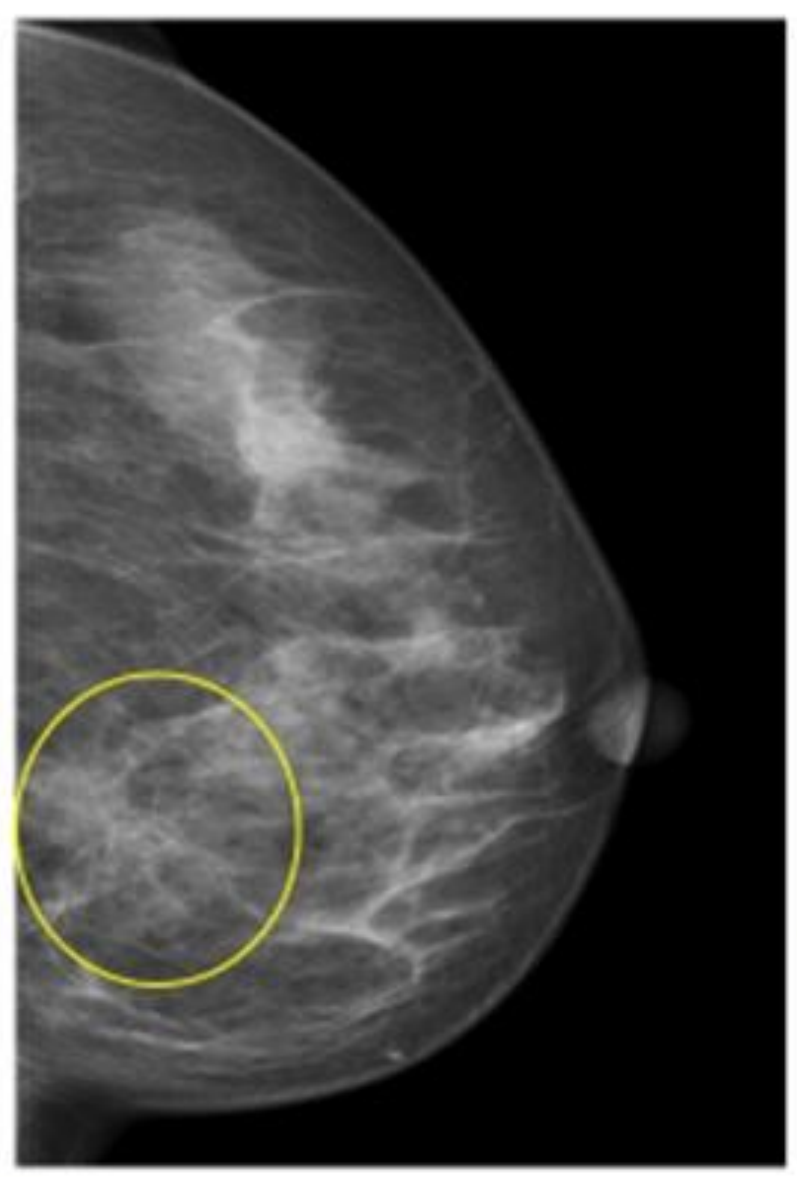

FIGURE A - DM

Left breast - CC view

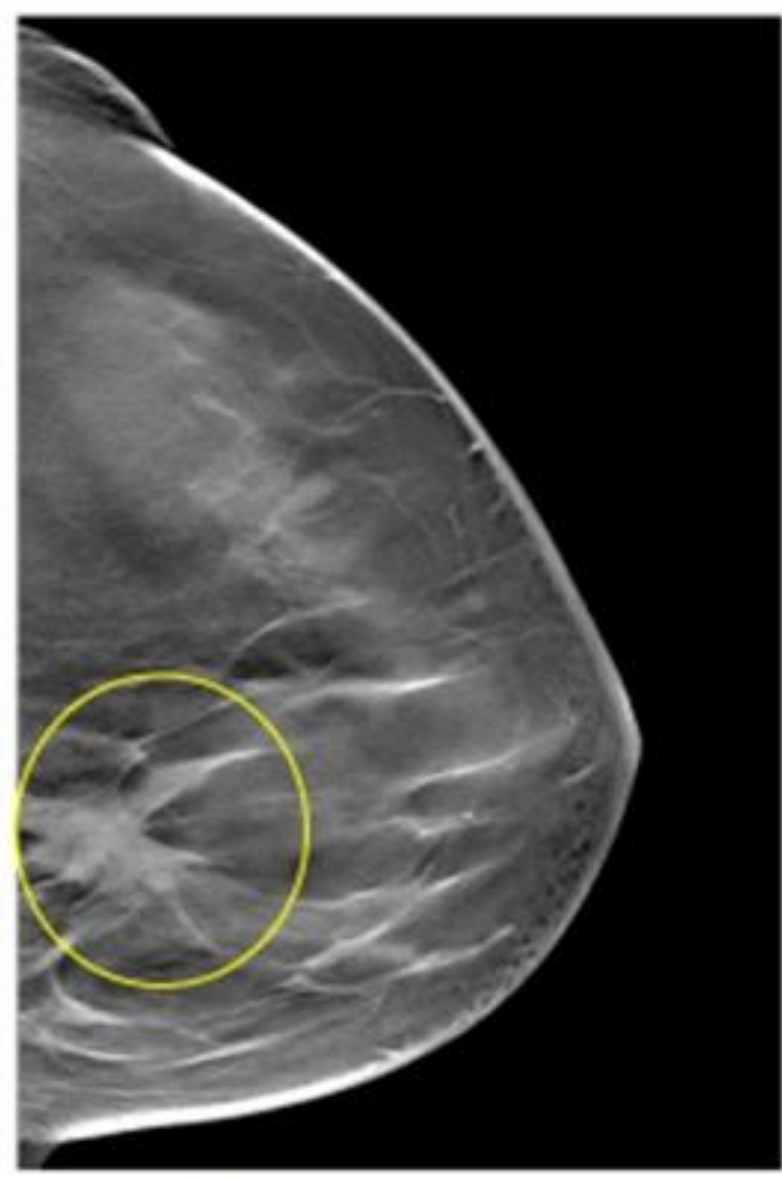

FIGURE B - DBT

Left breast - CC view 37-year-old female mammography CC views: ACR C.

DM: Left inner central region area of architectural distortion - BIRADS 3.

DBT: High density lesion in inner central region. The margin of the lesion appeared speculated on 3D digital tomosynthesis images (BIRADS 4). The tomosynthesis has better margin characterization and nullified the superimposition effect caused by normal breast parenchyma on the lesion which easily detected the spiculated margin and high density of this lesion and upgraded the BIRADS category from 3 to 4 . Ultrasoundconfirmed speculated borders. Pathologically proved to be invasive ductal carcinoma. 
Figure 3

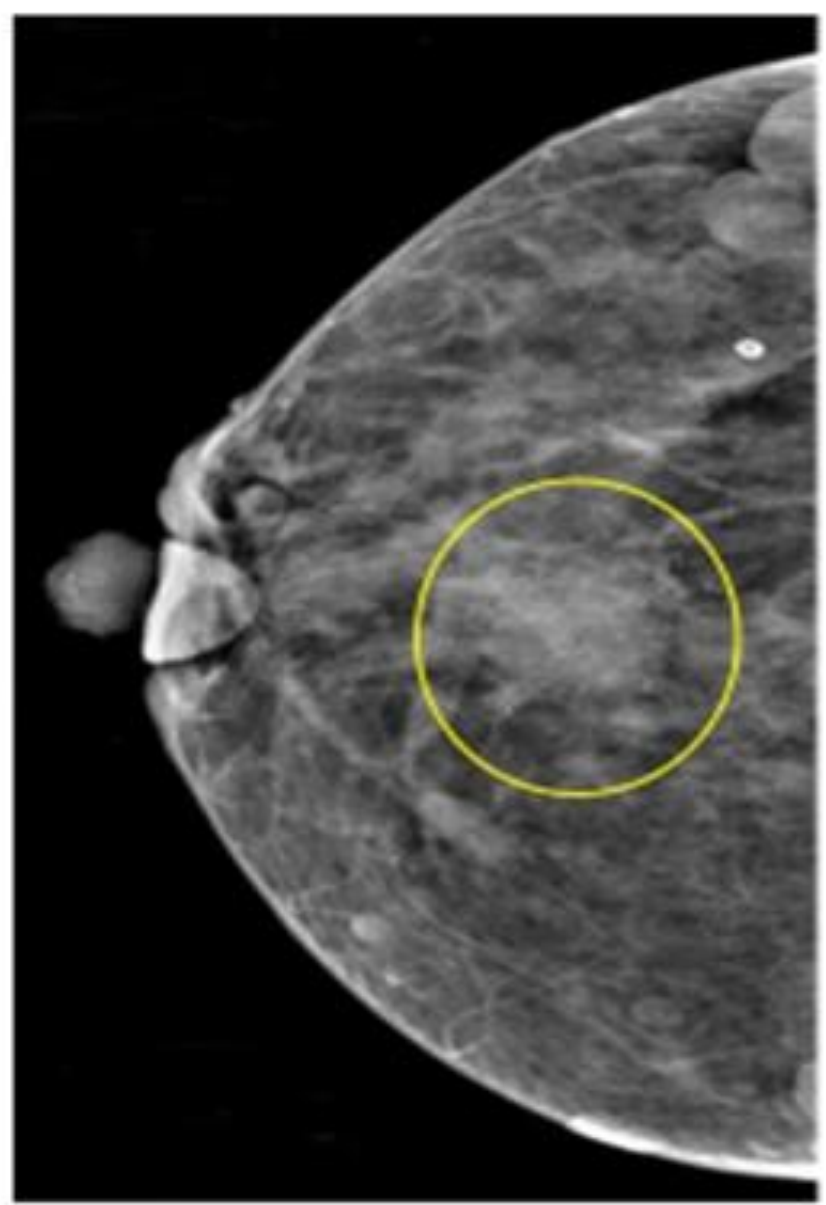

FIGURE A - DM

Left breast - CC view

50- year-old female. Mammogram CC views: ACR C.

DM: A high density mass in central region of the left breast with almost completely obscured margins - BIRADS 3.

DBT: 3D digital breast tomosynthesis showed left central rather well-circumscribed dense lesion BIRADS 2. 3D digital breast tomosynthesis easily detected the lesion and differentiated its margins from the overlying parenchyma and this changed the BIRADS from 3 to 2, proved by US to be cyst. All the lesions assigned BIRADS 1 and 2 were subjected to ultrasonography examination to confirm their benign nature. All the lesions (135/135) assigned BIRADS 1 and 2 on DBT turned out to be benign lesions on USG.

All the lesions assigned BIRADS 3 and 0 were subjected to ultrasonography examination to

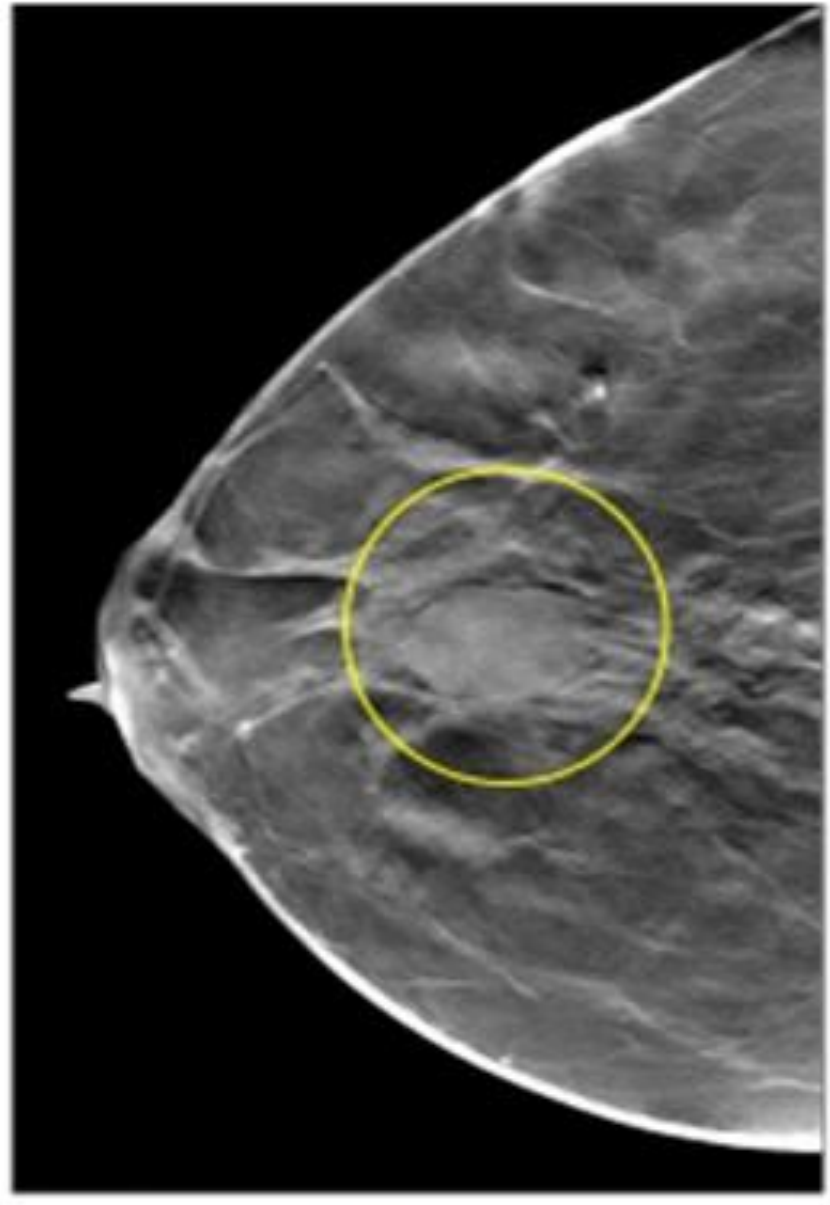

FIGURE B - DBT

Left breast - CC view

confirm their true nature and the suspicious lesions were subjected to HPE examination. Out of 29 lesions assigned BIRADS 3 and 0 on DBT, 21 lesions were found to be cysts, 5 lesions were found to be fibroadenoma and 3 lesions were found suspicious. This suspicious lesions were biopsied and all the lesions proved to be fibroadenoma on HPE.

All the lesions assigned BIRADS 4 and 5 were subjected to USG and HPE examination to confirm their malignant nature. Out of 61 DBT assigned BIRADS 4 and 5 lesions, 48 proved to be Invasive ductal carcinoma, 6 proved to be Lobular carcinoma, 4 proved to be Medullary carcinoma and 3 proved to be predominant Intraductal carcinoma.

$($ Benign $=$ BIRADS 1 and 2 , Malignant $=$ BIRADS 4 and 5) 


\section{BIRADS Classification}

$77.8 \%$ of BIRADS 3 and 0 lesions detected by 2D digital mammography (102/131) changed their category after 3D DBT and $22.2 \%(29 / 131)$ of digital mammography assigned BIRADS 3 and 0 lesions did not change after 3D DBT.

$77.8 \%$ percent of the lesions changed their BIRADS system classification following DBT:

1. 3D DBT upstaged to BIRADS 4 and 5 in 34 lesions representing $15.1 \%$ of the cases.

2. 3D DBT down staged to BIRADS 1 and 2 in 68 lesions representing $51.9 \%$ of the cases.

We performed a biopsy in all suspected findings on tomosynthesis. Of 225 patients, 60 were proven to have malignancy. Of this 60 patients, 46 patients had a single lesion, 6 had multifocal disease, and 8 had multicentric disease.

\section{Discussion}

Mammography is the most widely used imaging tool for screening of breast lesions. Breast cancers are detected mammographically by the looking for architectural distortion, asymmetry, and calcifications and associated features which may present as mass.

However, an important limitation of mammography is the masking of radiological signs of malignancy in the dense parenchyma as it is two-dimensional (2D) imaging. The overlapping fibroglandular tissues can either mimic a cancer or render an accurate benign diagnosis difficult, reducing specificity and the fibroglandular tissues can reduce conspicuity of abnormalities, reducing sensitivity ${ }^{[3]}$.

Solutions to these problems include further evaluation of the patient with ultrasound (US) or magnetic resonance imaging (MRI), but they are not the optimal solution as a screening tool because despite US being safe and inexpensive, there is increased operator-dependence and less interobserver agreement, particularly for small malignancies. MRI is comparatively safe and more sensitive, but it is expensive, timeconsuming and may not always be available in the same department ${ }^{[3]}$.
One way to improve the sensitivity and specificity of imaging is to perform three-dimensional (3D) imaging - DBT, which can reduce the confounding effects of fibroglandular tissues on cancer detection, especially in denser breasts.

Digital breast tomosynthesis (DBT) is a recent addition to the equipment used for digital mammography in which the breast is compressed, as with conventional 2D mammography, between a compression paddle and the detector housing. While the breast is kept stationary, the X-ray tubeis moved, usually in an arcuate motion, and a set of low-dose 2D images known as projections is collected. The projection images are then reconstructed into thin slices of $1 \mathrm{~mm}$ thickness each, which minimizes the superimposition effect of overlapping tissue and helps in detection of even subtle abnormalities ${ }^{[4]}$.

It has a highly promising role in increasing the sensitivity and specificity of digital mammography.

This study was undertaken to determine the sensitivity and specificity with predictive values for DBT at the Breast Imaging Unit of our hospital.

This study showed a high sensitivity and specificity in detecting malignant breast lesions by DBT as compared to that by DM.

This advantage of DBT over DM provides two main benefits -improved cancer detection rates and reduction in rates of call back.

\section{Advantages of 3d- DBT over DM:}

- Because of the reduced tissue overlap and structure noise on DBT, shape and density of the lesions are better visualized, with improved marginal analysis of mass lesions. This leads to more confident readings and allows the more accurate evaluation of lesions with accurate BIRADS categorisation of the lesions ${ }^{[1,4]}$.

- DM imaging has limitations, especially in heterogeneously dense breast tissue. True lesions can be obscured by superposition of normal breast parenchyma, 
i.esuperimposition artefact and summation artefactof normal breast parenchyma can simulate a true lesion. DBT is very helpful in the work-up of DM- detected abnormalities $^{[1,4]}$.

- DBT, compared with DM, allows better differentiation of the characteristic features defining the benign and malignant nature of the lesions; BI-RADS upgrade and downgrade is possible on DBT.

We aimed to detect and prove the improved capability of 3D digital tomosynthesis in evaluation of DM assigned BIRADS 3 and 0 breast lesions.

In our study, $77.8 \%$ of the BIRADS 3 and 0 lesions changed their BIRADS by 3D DBT 51.9 $\%$ were down staged to BIRADS 1 and 2, and $15.1 \%$ were "upstaged" to a higher BIRADS.

$22.2 \%$ of the BIRADS 3 and 0 lesions did not change their BIRADS.

Thus, 3D DBT significantly reduced the need for additional time-consuming imaging such as additional mammographic views or sonomammography thereby increasing the efficacy of the test by reducing the additional radiation dose, time, and money. DBT provided a great benefit of reducing patient's anxiety by avoiding unnecessary recalls ${ }^{[1]}$.

We found that DBT produced a significant change of BI-RADS category in $77.8 \% \mathrm{DM}$ assigned BIRADS 3 and 0 lesions with an upgrade in 15.1 $\%$ lesions (98.3\% were malignant) and a downgrade in $51.9 \%$ lesions $(97.05 \%$ were benign) in comparison to the DM.

However, these benefits come at the cost of increased radiation dose as compared to that in DM.

In spite of all this advantages, DBT also has a few disadvantages - There is a possibility that some of the malignances may be missed or misinterpreted in extremely dense breasts and increase in radiation dose as compared to that in DM. But, the increased total dose is still below the Food and
Drug Administration safety limits of $3 \mathrm{mGy} / \mathrm{view}$ [5].

In addition, further research is required to explore the optimal imaging technique that will give the best image quality with the least radiation dose to the patient. This can help in better patient diagnosis and improve further patient management. Finally, additional research is still needed to determine whether DBT can replace $\mathrm{DM}$ as a diagnostic technique and whether early detection of breast cancer using DBT is associated with decrease in mortality rate ${ }^{[5]}$.

The limitations of this study is its small sample size.

\section{Conclusion}

3D DBT significantly reduced the need for additional mammographic views and frequent follow-up studies as it gave better characterization for all BIRADS 3 and 0 lesions. 3D DBT did not show any false-negative results in this study and it did not miss any cancers. In addition, reduction in false positive results reduced the stress levels in women $^{[1]}$. Therefore, 3D digital breast tomosynthesis should be applied in the diagnostic algorithm in patients with mammography detected BIRADS 3 and 0 lesions

The above findings are concordant with that of many previous researches, which have established that DBT increases the sensitivity and specificity of DM. Consequently, In light of our data, and considering the high diagnostic performance of DBT, we recommend the use of DBT as an additional imaging modality to improve diagnostic accuracy in detecting and characterizing indeterminate breast ilesions.

\section{Abbreviations}

BIRADS -Breast imaging-reporting and data system, DBT - Digital breast tomosynthesis, DM Digital mammography, 2D- Two Dimensional

\section{Statement of Ethics}

Our study protocol was approved by the Ethics Committee of our institution. 


\section{Consent for participation and publication}

Objectives and methodology were clearly explained to the patients, and all patients provided written informed consent. If the patient was unconscious or deceased, consent for publication was requested, written informed consent for the publication of this data was given by their parent or legal guardian.

\section{Competing Interests}

The authors declare that they have no competing interests.

\section{References}

1. Galati F, Marzocca F, Bassetti E, Luciani ML, Tan S, Catalano C, Pediconi F. Added Value of Digital Breast Tomosynthesis Combined with Digital Mammography According to Reader Agreement: Changes in BI-RADS Rate and Follow-Up Management. Breast Care (Basel). 2017 Sep;12(4):218-222. doi: 10.1159/000477537. Epub 2017 Aug 29. PMID: 29070984; PMCID: PMC5649241.

2. American College of Radiology: ACR BIRADS ${ }^{\circledR}$ Atlas 5th Edition. ACR, 2013.

3. Ali, E.A., Adel, L. Study of role of digital breast tomosynthesis over digital mammography in the assessment of BIRADS 3 breast lesions. Egypt J Radiol Nucl Med50, $48 \quad$ (2019). https://doi.org/10.1186/s43055-019-00525

4. Martin Sonnenschein, Christian Waldherr. Introduction to Tomosynthesis. In: Illustrated. Atlas of Breast Tomosynthesis: Imaging Findings and Image-Guided Interventions. Springer, 2017.

5. Asbeutah AM, Karmani N, Asbeutah AA, Echreshzadeh YA, AlMajran AA, AlKhalifah KH. Comparison of Digital Breast Tomosynthesis and Digital Mammography for Detection of Breast Cancer in Kuwaiti Women. Med PrincPract. 2019;28(1):10-15. doi:
10.1159/000495753. Epub 2018 Nov 26.

PMID: 30476905; PMCID: PMC6558339. 